\title{
Revisiting Client/Server Computing
}

Carl S. Guynes, University of North Texas, USA

John Windsor, University of North Texas, USA

\begin{abstract}
The role of the Information Technology department is changing as it continues to mature and adapt to changes in technology, much of that change dealing with its interaction with corporate end-users. IT departments must continue to move in a more user friendly direction or they are going to encounter serious political problems with top management. The increased interest in cloud computing, green computing, and in outsourcing are further indicators of the problems that IT departments are currently facing. Information Technology departments must approach client/server with a clear view of the lasting business benefits that it can bring rather than opposing or resisting the migration
\end{abstract}

Keywords: Corporate Politics; Client/Server Computing; Cloud Computing

\section{INTRODUCTION}

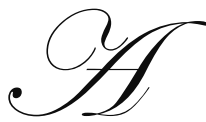

political situation is continuing to evolve in "Corporate America" that significantly impacts corporate Information Technology (IT) departments. The IT departments are feeling increased pressure from the end-user community to provide them with even more control over their data and the ability to run the software of their choosing on their own workstations. For example, recent advances in mobile computing have made it possible for customers carrying handheld devices to have access to data and information services regardless of their physical location. Customers expect the same level of service in terms of availability and performance from the mobile applications as with their non-mobile counterparts. Different types of client/server computing architectures are used today that facilitate such mobile access of data. In order to achieve high performance and availability, replicas of data servers are usually added to tolerate failures and balance workloads.

The role of the Information Technology department is changing as it continues to mature and adapt to changes in technology, much of that change dealing with its interaction with corporate end-users. IT departments must continue to move in a more user friendly direction or they are going to encounter serious political problems with top management. The increased interest in cloud computing, green computing, and in outsourcing are further indicators of the problems that IT departments are currently facing.

One reason that client/server computing is still popular is because end-users believe that IT departments are not responsive enough to their needs. The reason that client/server computing is still growing in popularity is because top management believes that it will save them money, and end-users believe that it will solve many of their computing problems. Unfortunately, neither of the above is necessarily true. A major problem is that neither top management nor end-users fully understand all that is involved in corporate computing. Top management can easily understand that the hardware for a client/server system costs less than a mainframe, but they have a difficult time understanding multiplatform software complexity and costs, or understanding the difficulty of maintaining controls that protect data integrity. The typical end-user still has little or no understanding of why they cannot upload their local data to the primary corporate database. Generally, end-users want complete access to corporate databases and are not particularly concerned with such issues as access controls and data integrity and privacy considerations.

The IT department is committed to protecting the systems and data that are the lifeblood of the organization. IT management is concerned with where corporate data are being downloaded and with the stability of the networking systems that are installed throughout the organization. They continue to be concerned with maintaining the integrity of the corporate database and maintaining extensive controls to protect the data. IT management views client/server systems as presenting additional problems to an already complex situation. 
It is the purpose of this article to suggest some ways to implement client/server systems that will be satisfactory to top management, end-users, and the Information Technology department. We attempt to define several terms associated with client/server computing, present the positives that can be attained from a successful implementation, and address some of the problems associated with client/server computing.

\section{CLIENT/SERVER DEFINITIONS}

Even though client/server is still evolving as a form of computing, there are some fundamental concepts in place. The distinguishing feature of client/server is that it contains cooperative processing capabilities that physically split the processing performed by the client from that performed by the server while presenting a single logical picture to the user. Client work-stations need to be intelligent because they must have processing capabilities and make requests to servers. Servers perform a set of special tasks for any other device requesting their services. Networks connect the work-stations and servers together. Software applications tie the components together to form the client/server architecture.

The client addresses the server by way of queries, or commands, and waits for appropriate responses from the server. Depending on the specific implementation, the queries can be performed synchronously or asynchronously. Upon receipt of the data from the server, the client can then perform data analysis and presentation locally. In a heterogeneous client/server environment, there are several operating systems the client can run under, including Windows and UNIX. The workstation provides the client portion of the model enabling the user to manipulate data received from the server.

The main force behind client/server computing is the empowerment of the end-user by delivering information and processing capabilities to the desktop of the users. In the client/server environment, the graphical user interface (GUI) is the front end to the network. Users are not network experts, nor should IT expect them to be. Refined interfaces have simplified what can otherwise be complex systems, enabling the user to focus on the job at hand.

A server is a process, or set of processes, which reside on a machine to provide services to one or more clients. The server is connected to the LAN and is made available to clients. The nature of the server depends upon the goal of the client/server system. The server could be anything from a print server (low machine power needed) to a file server (medium machine resources needed) to a database server (high amount of machine resources needed). The server does not initiate contact with the clients, but instead monitors the network waiting for requests from clients. Once a request is received, the server processes the request and returns an answer. Ideally, the servers hide the complex client/server system from the client and the user, and ideally, the user should not be aware that there is a client/server relationship. Servers must be able to grow to support growing numbers of clients as needs arise. To the clients, a server must be just that - a server. The underlying hardware platform and operating system must be transparent to the clients. The clients are only interested in what the server does. For example, if a database server uses an SQL interface, the clients do not need to know if the server is running UNIX, Windows, or any other operating system. The client should only be concerned with the SQL interface.

In many large corporations that have data residing on a mainframe computer, it will be necessary for the server to provide a gateway to the mainframe for the clients. It is very important for the server to have adequate disk space to serve the clients, since in many client/server systems, the server is the central location for data. The client portion and the server portion usually operate on separate computer platforms, but they do not have to. It is occasionally possible for a client and server to operate on the same hardware platform. For example, a client to one server may be a server to another client. However, to qualify as true client/server computing the ability to install the client and server on two separate platforms must be present. Either the client or the server hardware platform can be upgraded without having to upgrade the other platform. You must be able to change one of the nodes in the client/server system without having to change the other nodes. Each node is independent of the other nodes with the server able to service multiple clients concurrently.

\section{BENEFITS FROM CLIENT/SERVER}

Client/server computing plays an important role in transferring large, centrally controlled mainframe applications to the smaller, distributed systems. Because of the enormity of many mainframe applications, the distributed processing that client/server computing brings helps distribute the resources necessary to implement these systems. A 
common distributed system architecture style being used is the three-tiered client/server style. This client/server architecture incorporates an active replication strategy to address reliability and performance concerns. When installed properly, client/server can reduce the operating costs of information systems departments because the hardware typically requires a less sophisticated support environment.

Client/server should provide a better return on technology investments because it allows niche or specialized technology to be configured as "common resources," which are widely available within the computing environment. If the proper controls are in place, client/server computing should allow greater access to corporate data and information.

In the client/server environment, the workstation controls the user interface. All user interface commands are processed on the client. This frees the server to do other types of computing. As the server is free of user interface issues and other types of computations performed by the client, the server's resources are left to do other computing, such as intensive number crunching or large database searches. Full data files do not need to be sent to the workstation for processing as the data is stored and manipulated on the server. Only answers to requests from the clients are sent. As the user interface is controlled at the client, user keystrokes are not sent through the network. Both of these dramatically cut down on network utilization. Client/server systems allow you to put applications on less expensive workstation servers instead of expensive mainframe and mid-range systems. Existing mainframes are still used as enterprise data management and storage systems, with much daily activity moved to lower-cost servers. Software independence from hardware platforms is an important feature of growing significance, given the emergence of new distributed computing paradigms. Server performance is also cheaper than equivalent mainframe performance. Server MIPS can provide a cost advantage of several hundred to one compared to mainframe MIPS. Another cost advantage is that client/server database management systems are less expensive than mainframe DBMS. Client/server systems can speed up the application development process because the developers do not have to compete for mainframe resources. This provides faster response time because of fewer bottlenecks and may have an impact on reducing the systems development backlogs found in most companies.

Workstations can run applications that are impractical to run on the mainframe. CPU-intensive applications, such as graphics and data analysis, require client/server's distributed processing and shared data. By running these applications on workstations, the server can be freed up to process other applications. The response time to reply to complex queries on a mainframe can sometimes take longer than on a client/server system. Generally, the client/server model provides faster performance for CPU-intensive applications because the processing is done locally and does not have to compete for mainframe CPU time.

Client/server computing allows multi-user access to shared databases. Database servers centralize the data which permits remote access to the data. With client/server computing, users can access the data that was historically stored in their departments and can access other corporate data as needed. With client/server computing, users have access to large databases, printers, and high speed processors, all of which will improve user productivity and quality. Since the client receives only the data requested, network traffic is reduced and performance is improved. Access is also easier because resources are transparent to users.

IT must be aware that storage systems using hierarchical directory trees do not meet scalability and functionality requirements for the exponentially growing datasets and increasingly complex queries in Exabyte-level systems with billions of files. They should be looking into semantic-aware organizational methods which use metadata semantics of files to aggregate correlated files into semantically similar groups. This new decentralized design improves system scalability and reduces query lag-times for complex queries in large-scale file systems.

The Client/Server model has well defined data security and assurance standards. Proper adherence to these standards provide for controlled restrictive access to sensitive information. Use of these standards and restrictions on processing rights also provide for improved data integrity. These standards also provide for defense in layers, the current best practice for providing electronic protection for both sensitive corporate data and critical computing resources.

Client/server computing allows organizations to extend the lifespan of existing computer equipment. The existing mainframes can be utilized to perform as servers and to process many of the existing applications that cannot be 
converted to client/server applications. The user's workstation can be kept to act as front-end processors. Client/server computing is flexible in that either the client or the server platform can be independently upgraded at any time. As processing needs change, servers can be upgraded or downgraded without having to develop new front-end applications. As the number of users increases, client machines can be added without affecting the other clients or the servers.

\section{PROBLEMS ASSOCIATED WITH CLIENT/SERVER}

Companies moving more applications from a mainframe environment to client/server computing face several issues. Client/server technology requires significant technical support as it allows a company to use hardware and software from many different vendors. Support contracts are required with each of these vendors. In order to minimize the difficulties of having multiple vendors, a company must establish internal hardware and software standards. The full-scale implementation of client/server computing requires either reorganizing existing departments or creating new ones. Most developers understand either the mainframe system or PC systems, but not both. Since there is a shortage of skilled and experienced developers, there is more trial and error in developing client/server applications than for older well understood mainframe applications. Developers continue to need to go through extensive training to learn the technology. In addition to retraining current employees, a company may well need to hire specialist in LAN administration, database administration, application development, project management, and technical support for users.

As with most developing technology, client/server computing does not have agreed upon industry standards. Currently, there is no standard way of retrieving, manipulating, and maintaining complex data like graphics, text, and images. Standards must be established in order for client/server to allow the use of products from different vendors. SQL is a standardized data access language; however, each DBMS vendor has its own SQL dialect. This adds to the complexity of building transparent links between front-end tools and back-end database servers.

There are serious security and access control issues to be considered. Since the server is usually the central location for critical data, adequate physical security and operational security measures need to be taken to insure the safety of the data. Backup and recovery procedures are improving, but logging procedures are still lacking. There are a large number of tools to perform security and control functions on mainframe systems. Many of these tools can help with the client/server effort, but not all are designed specifically for client/server computing. The lack of comprehensive automatic backup and recovery tools is another problem. Companies must be careful as to which mission critical applications they let reside on a client/server system.

Implementing Client/Server computing in a Cloud Computing environment increases the security and access control issues. Access to the "Server" is from anywhere in the world. Failure to require strong authentication procedures starting with strong passwords, and failure to patch clients, as well as the servers, will virtually guarantee the failure of system security.

Performance monitoring and capacity planning tools are a low priority for many client/server administrators. It can be less expensive to buy a new server and add it to the system than to spend resources on capacity and performance analysis. Not all tools necessary to support client/servers are fully developed. There is still a lack of robust client/server oriented communications, diagnostic, and applications tools. These troubleshooting tools are more powerful than they were several years ago, but they are less robust than those readily available for mainframes. There is also a lack of robust tools for converting existing applications to client/server routines. This forces client/server users to either write new client/server applications from scratch or use the existing applications on the mainframe. As companies have invested in millions of lines of mainframe code that is not practical to convert to client/server, mainframes will continue to be a major asset for high volume applications.

\section{IMPLEMENTATION CONSIDERATIONS}

The successful implementation of client/server systems requires involvement by top management, representatives from user groups, and key IT representatives. It is possible for the IT group to become so involved with the technical decision/selection process that it ignores the business's strategic concerns. Business users may not see the value of careful and reasoned selection and end up with a solution that may seem to meet their requirements, but may not 
solve their long-term needs. A major issue to be considered should include ways that the new or upgraded systems could improve efficiency, reduce costs, provide a competitive advantage, and reduce cycle time. IT should build a model of the organization's work flow and data flow. This will help in designing networks and will help determine how data should be distributed.

There should be careful consideration of the utilization of front-end tools. Three front-end tools, in particular, are SQL-based tools, user interface builders, and integrated development tools. The tools that are picked will be determined by the data and systems requirements, and will require a great deal of investigation to decide which application will be right for the system's particular needs.

Training is another key to the successful implementation of a client/server solution. Without proper training, the risk of failure is high. If IT personnel are not aware of the fundamental limits of current client/server technology, they can either exceed or under-utilize the limits of the system which would seriously damage the success of the project. Other key decisions that must be made before implementing a client/server system are:

1. What operating systems will be used?

2. What Graphical User Interface will be used?

3. What hardware platforms will be used?

4. What online transaction processing systems will be used?

In client/server computing, no answer is perfect. Technology that is selected now may not be the solution in the future. IT managers need to realize that some custom components may have a limited lifetime. Organizations moving from the PC environment to the client/server model will be required to invest in a database administrator and a network administrator to be able to manage the centrally-controlled database. Client/servers will also require more support from vendors and for users. With the new technology, organizations will have to invest in vendor support programs to receive assistance.

\section{CONCLUSIONS}

The changes occurring in the IT environment are the result of the increasing desire to move the technology beyond its present state. With the availability of new and cheaper personal technologies, the drive for direct exploitation of available information is increasing. Information Technology departments should approach client/server with a clear view of the lasting business benefits that it can bring rather than opposing or resisting the migration. What is required to accommodate this change is for the IT group to recognize that its responsibilities have shifted to the establishment and management of infrastructures, services and data quality. It is no longer appropriate for IT to position itself between the end-user and the technology. Most of the issues presented by client/server systems are similar to those presented by mainframe computing. For this reason IT is uniquely positioned to lead in the correct implementation of client/server systems.

The users must be made aware of the client/server technology and the benefits that it can provide, and knowledge of the proposed client/server solution should be disseminated to all concerned groups. This knowledge is key to the proper use of the technology, as the users are in a better position to evaluate the technology. If the users can use the system and it solves the business problem, the chance for acceptance is high. Top management, end-users, and the IT group must work together to solve the types of business problems for which client/server is best suited.

\section{AUTHOR INFORMATION}

Dr. Carl S. Guynes is a Regents Professor of Information Systems at the University of North Texas. He received a doctorate in quantitative analysis from Texas Tech University. Dr. Guynes' areas of specialization are client/server computing, end-user computing, data administration, and information resource management. His most recent research efforts have been directed in the areas of client/server computing and data administration. Some of the journals in which Dr. Guynes has published include Communications of the ACM, Information \& Management, The Journal of Information Systems Management, Journal of Accountancy, Journal of Systems Management, The Journal of Database Management, 
The CPA Journal, The Journal of Computer Information Systems, Information Strategy, Computers and Security, and Computers and Society. Guynes@unt.edu

Dr. John C. Windsor is a professor of Information Systems and former Director of the Information Systems Research Center at the University of North Texas. He received his Ph.D. in Decision Sciences from Georgia State University. He has published six books and over 60 articles in such journals as Data Base, IIE Transactions, Information \& Management and Computers \& Security. His research interests include software and data engineering, systems security, collaborative computing, and the organizational impact of information technology. Windsor@unt.edu

\section{REFERENCES}

1. Das , Olivia and Das, Arindam. "Performability evaluation of mobile client/server systems." Proceedings of the 200 ACM symposium on Applied computing. (March2008):2197-2201.

2. Hua, Yu, Jiang, Hong, Zhu, Yifeng, Feng, Dan, Tian, Lei. "SmartStore: a new Metadata organization paradigm with semantic-awareness for next-generation file Systems."Proceedings of the Conference on High Performance Computing Networking, Storage and Analysis. (November 2009): 43-59.

3. Lai, Albert and Nieh, Jason. "On the performance of wide-area thin-client computing." ACM Transactions on Computer Systems. Vol 24 , No 2, (May 2006):175-209.

4. $\quad$ Ryan, Hugh. "Issues in Client/Server Development." Information Systems Management.(Fall 1992): 48-50.

5. $\quad$ Sinha, Alok. "Client/server Computing." Communications of the ACM. Vol 35, No 7, (July 1992): 77-98.

6. Toegl, Ronald, Winkler, Thomas, Nauman Mohammad, and Hong, Theodore. "Towards platformindependent trusted computing." Proceedings of the 2009 ACM workshop on Scalable trusted computing. (November 2009): 61-66.

7. Zarandioon, Saman, Yao, Danfeng, and Ganapathy, Vinod. "Privacy-aware Identity management for clientside mashup applications." Proceedings of the $5^{\text {th }}$ ACM workshop on Digital identity management. (November 2009): 21-30.

8. Zenarosa, Gabriel and Simanta, Soumya "Experiences In engineering active replication into a traditional three-tiered client/server system." Proceedings of the 2008 RISE/EFTS Joint International Workshop on Software Engineering for Resilient Systems. (November 2008): 55-60. 